\title{
Erratum: Large Deviation Principle Linking Lineage Statistics to Fitness in Microbial Populations [Phys. Rev. Lett. 125, 048102 (2020)]
}

\author{
Ethan Levien®, Trevor GrandPre®, and Ariel Amir@ \\ (Received 21 December 2020; published 19 February 2021)
}

DOI: 10.1103/PhysRevLett.126.079901

In our Letter, we presented an approach to linking the statistics of independent lineages to a population's fitness. Here we correct two minor errors and point out an inconsistency with the algorithm used to generate some of the figures and the one described in the Letter. These errors have no implications on the main conclusions of our work.

We first point out two errors in the Letter. Before Eq. (10), we state that $I^{\prime \prime}\left(\langle\gamma\rangle_{p}\right) \gg 1$. The correct condition for Eq. (10) to hold is $I^{\prime \prime}\left(\langle\gamma\rangle_{p}\right)\langle\gamma\rangle_{p} \gg 1$. Second, in the Supplemental Material following Eq. (S28) [1], we state that $K_{T}$ grows as $\sqrt{T}$. The correct statement should be that $K_{T} / T$ scales as $\sqrt{T}$, thus $K_{T}$ scales as $T^{3 / 2}$. Note that the scaling of $K_{T}$ was not used elsewhere in the Letter.

We now turn to a distinction between the estimator used in our simulations and the one described in the Letter. As described in the main text of the Letter, our estimator of the population growth rate $\Lambda$ [given in Eq. (3) of the main text] takes the form

$$
\Lambda_{\operatorname{lin}}=\frac{1}{T} \ln \left[\frac{1}{M} \sum_{j=1}^{M} 2^{n_{j}}\right],
$$

where $n_{j}$ is the number of divisions among the $j$ th lineage in an ensemble of $M$ lineages. If one observes 100 cells on a lineage, the number of divisions should be $n_{j}=99$. However, for our simulations and data analysis, we used the number of cells instead of the number of divisions. If $\tilde{\Lambda}_{\text {lin }}$ is the estimator obtained from the number of cells, the relationship between $\tilde{\Lambda}_{\text {lin }}$ and $\Lambda_{\text {lin }}$ is given by

$$
\tilde{\Lambda}_{\operatorname{lin}}=\Lambda_{\operatorname{lin}}+\frac{1}{T} \ln (2) .
$$

Because the difference between the estimators scales as $1 / T$, all of the main results of our Letter concerning asymptotic properties of $\Lambda_{\text {lin }}$ apply to $\tilde{\Lambda}_{\text {lin }}$. Specifically, both estimators will converge to $\ln (2)\langle\gamma\rangle_{p}$ at $T \rightarrow \infty$ for fixed $M$ and converge to the true population growth rate if the large $T$ limit is taken after taking the limit $M \rightarrow \infty$.

We thank Arthur Genthon (ESPCI) and David Lacoste (ESPCI) for bringing our attention to this distinction, which explains a discrepancy between Fig. 2(a) of their paper [2], where the convergence of $\Lambda_{\text {lin }}$ is from below, and our Figs. 3 (inset), S3 (inset), and S4, where the initial convergence is from above. Note that $\operatorname{err}\left(\hat{\Lambda}_{\text {lin }}\right)$, which is defined in Eq. (12) of our Letter and plotted in the main panel of these figures, will obey the same nonmonotonic convergence regardless of whether cells or divisions are used.

[1] See Supplemental Material at http://link.aps.org/supplemental/10.1103/PhysRevLett.125.048102.

[2] A. Genthon and D. Lacoste, Fluctuation relations and fitness landscapes of growing cell populations, Sci. Rep. 10, 11889 (2020). 